\title{
Development and validation of Nasal Polyposis Quality of Life Questionnaire (NPQ)
}

\author{
Ilaria Baiardini ${ }^{1}$, Giovanni Paoletti ${ }^{2}$, Alessia Mariani ${ }^{2}$, Luca Malvezzi ${ }^{2}$, Francesca Pirola ${ }^{2}$, \\ Giuseppe Mercante $^{2}$, Giuseppe Spriano ${ }^{2}$, Francesca Puggioni ${ }^{2}$, Francesca Racca ${ }^{2}$, Giulio \\ Melone $^{2}$, Giacomo Malipiero ${ }^{3}$, Sebastian Ferri ${ }^{2}$, Giorgio Walter Canonica ${ }^{1}$, and Enrico \\ Heffler $^{3}$ \\ ${ }^{1}$ Humanitas University \\ ${ }^{2}$ Humanitas Clinical and Research Center, IRCCS \\ ${ }^{3}$ Humanitas Clinical and Research Hospital, IRCCS
}

September 24, 2020

\begin{abstract}
Background: To date, no disease-specific tool is available to assess the impact of Chronic Rhinosinusitis with Nasal Polyps (CRSwNP) on Health Related Quality of Life (HRQoL). Therefore, the purpose of this study was to develop and validate a questionnaire specifically designed to this aim: the Nasal Polyposis Quality of Life questionnaire -NPQ. Methods: According to the current guidelines, the development and validation of the NPQ occurred in two separate steps involving different groups of patients. Results: In the development process of NPQ an initial list of items of 40 items was given to 60 patients with CRSwNP; the 27 most significant items were selected and converted into questions. The validation procedure involved 107 patients (mean age 52.9 \pm 12.4 ). NPQ revealed a five-dimensional structure and high levels of internal consistency (Cronbach's alpha 0.95). Convergent validity (Spearman' coefficient $\mathrm{r}=0.75 ; \mathrm{p}<0.01$ ), discriminant validity (sensitivity to VAS score), reliability in a sample of patients with a stable health status (Interclass Coefficient 0.882 ) were satisfactory. Responsiveness to clinical changes was accomplished. The minimal important difference was 7. Conclusion: NPQ is the first questionnaire for the assessment of HRQoL in CRSwNP. Our results provide that the new tool is valid, reliable, and sensitive to individual changes.
\end{abstract}

\section{Hosted file}

manuscript.pdf available at https://authorea.com/users/361320/articles/482749-developmentand-validation-of-nasal-polyposis-quality-of-life-questionnaire-npq 


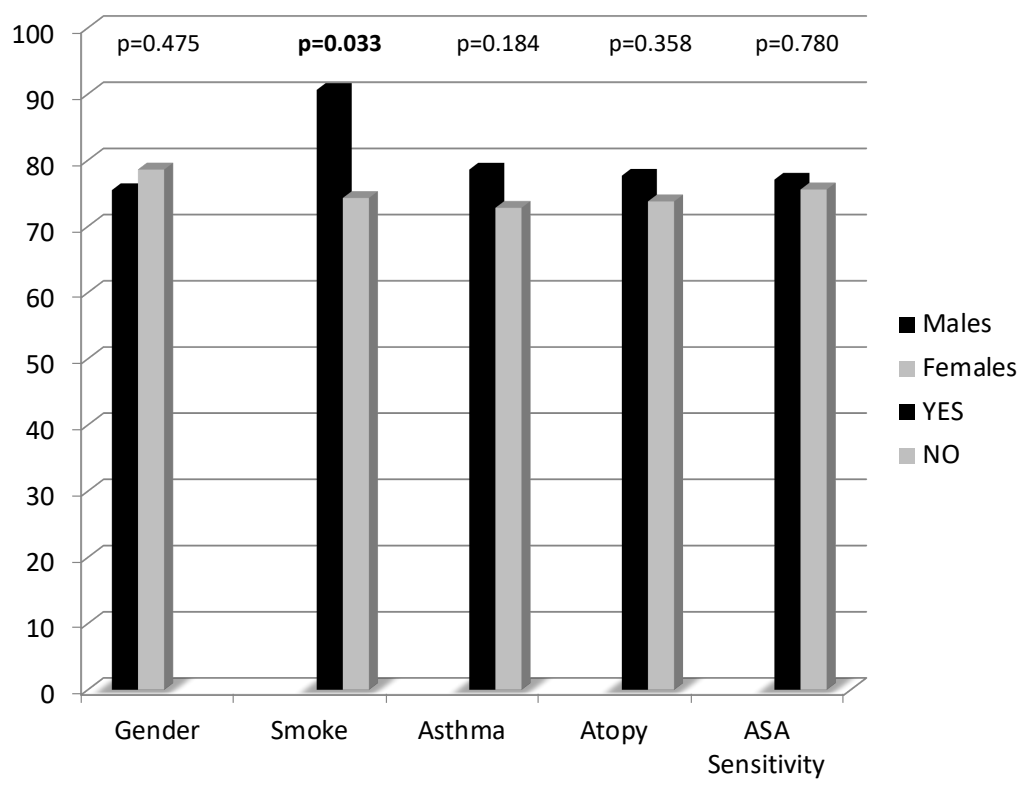

